to achieve sustained remission. The authors thus examined the immunomodulatory effects that MTX exerts on two major players in immune tolerance - regulatory (Tregs) and effector (Teffs) T cells.

Materials and methods Peripheral blood mononuclear cells (PBMCs) from 25 extended oligoarticular and polyarticular JIA patients were isolated before MTX start, 3 and 6 months after MTX start. The authors analysed frequency and phenotype of Tregs by flow cytometry. The function of Tregs was evaluated in CFSE-suppression assays. Proliferation of Teffs was examined in proliferation assays in the presence of anti-CD3. Teff cytokine production was measured ex vivo with flow cytometry upon PMA-ionomycin stimulation.

Results The frequency of FoxP3 ${ }^{+}$Tregs and the FoxP3 content per cell did not change upon MTX start. Suppressive capacity of Tregs appeared to be lower 6 months after MTX start compared to 3 months after MTX and prior to MTX start. In cross-over experiments, however, suppressive capacity of Tregs from all time points was equal. Teffs after MTX start proliferated significantly more in comparison to Teffs prior to MTX upon stimulation with anti-CD3. The authors observed an increase in IFN- $\gamma$ and TNF- $\alpha$ production.

Conclusions MTX does not exert immunomodulatory actions on Tregs. Instead, the authors observe changes in Teffs upon MTX start; they show that Teffs after MTX start have increased proliferation. The authors will explore the effect of MTX on Teffs further.

\section{A102 METHOTREXATE DOES NOT EXERT IMMUNOMODULATORY EFFECTS ON REGULATORY T CELLS BUT ON EFFECTOR T CELLS}

Maja Bulatovic, Sebastiaan Vastert, Wilco de Jager, Frederiek Verweij, Femke van Wijk, Nico Wulffraat, Berent Prakken Department of Pediatric Immunology, University Medical Center, Wilhelmina Children's Hospital, Utrecht, The Netherlands

\subsection{6/ard.2010.148981.5}

Background and objectives Methotrexate (MTX) is the most commonly used antirheumatic drug in juvenile idiopathic arthritis (JIA). MTX induces 'remission on medication' in $70 \%$ of patients and continuous 'remission off medication' in up to $50 \%$ of patients. MTX is unique in the realm of antirheumatic drugs since it is currently the only drug that appears capable of establishing immune tolerance in JIA. In spite of this, it is unclear which immunomodulatory mechanisms enable MTX 